\title{
Heartbeat: benefits of continued physical activity in late life
}

A heart healthy lifestyle includes regular physical activity. However, evidence supporting the benefits of exercise are mostly derived from relatively young populations with sparse data on whether exercise in the elderly is of continued value for prevention of adverse cardiovascular events. In order to better understand the potential benefit of physical activity in older adults, Barbiellini Amideli and colleagues ${ }^{1}$ examined the degree of physical activity, and change over time, in a cohort of over 3000 adults age 65 years or older with 20 year follow-up. Overall, physical activity was associated with a significantly reduced mortality risk for men (HR: 0.72 95\%CI 0.62, 0.84) and women (HR: 0.81 95\% CI $0.72,0.92$ ) (figure 1). However, the benefit of physical activity in reducing risk of coronary heart disease and heart failure was most evident in men with a high level of physical activity starting early in late life (eg, age 70-75 years).

In the accompanying editorial, Fabris and Sinagra $^{2}$ discuss the importance of physical activity in older adults both to increase longevity and to reduce adverse cardiovascular events along with attendant healthcare system costs. On a detailed analysis of the data, the effects of exercise were most evidence with only $20-40 \mathrm{~min}$ of exercise per day. In addition, exercise was most effective when started early (age 70 years) in late life, rather than late (age 80-85 years) in late life. Oddly, although risk reduction was noted for coronary disease and heart failure, there was no effect on stroke risk despite the shared pathophysiology of these conditions. Finally, Fabris and Sinagra ${ }^{2}$ point out that physical activity levels are related to several other factors that may contribute to cardiovascular risk (figure 2).

The potential long-term effects of combat-related traumatic injury (CRTI) on the cardiovascular system have received little study previously. In this issue of Heart, Boos and colleagues ${ }^{3}$ compared 579 adult male combat veterans (mean age 34 years) with CRTI matched to 565

Division of Cardiology, University of Washington, Seattle, Washington, USA

Correspondence to Professor Catherine M Otto, Division of Cardiology, University of Washington, Seattle, WA 98195, USA; cmotto@uw.edu
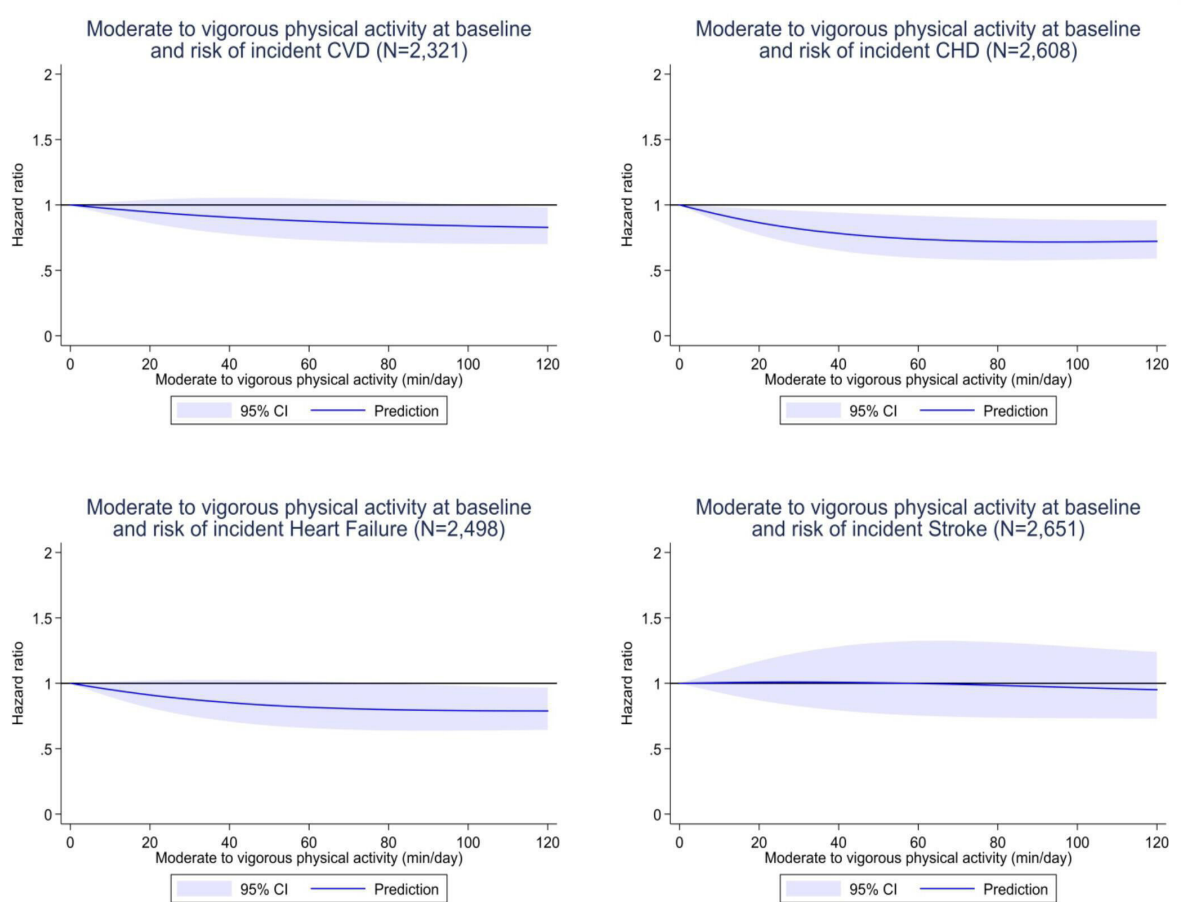

Figure 1 Association between moderate to vigorous physical activity a (min/day) at baseline and incident cardiovascular disease (CVD), coronary heart disease (CHD), heart failure and stroke. A physical activity (PA) was considered as (moderate PA min/day) $+2^{*}$ (vigorous PA min/day). All splines were adjusted for sociodemographic variables, stratified by categorised year of birth.

uninjured men at a mean of 8 years after deployment to Afghanistan. Metabolic syndrome was more common in men with CRTI compared with the uninjured group $(18.0 \%$ vs $11.8 \%$; adjusted risk ratio $1.46,95 \%$ CI 1.10 to $1.94, \mathrm{p}<0.0001)$. In addition, arterial stiffness was higher in men with CRTI, measured by heartrate adjusted central augmentation index. Other differences included a larger wait circumference, greater visceral fat area, higher triglyceride and lower high-density lipoprotein levels, increased insulin resistance and higher C-reactive protein levels

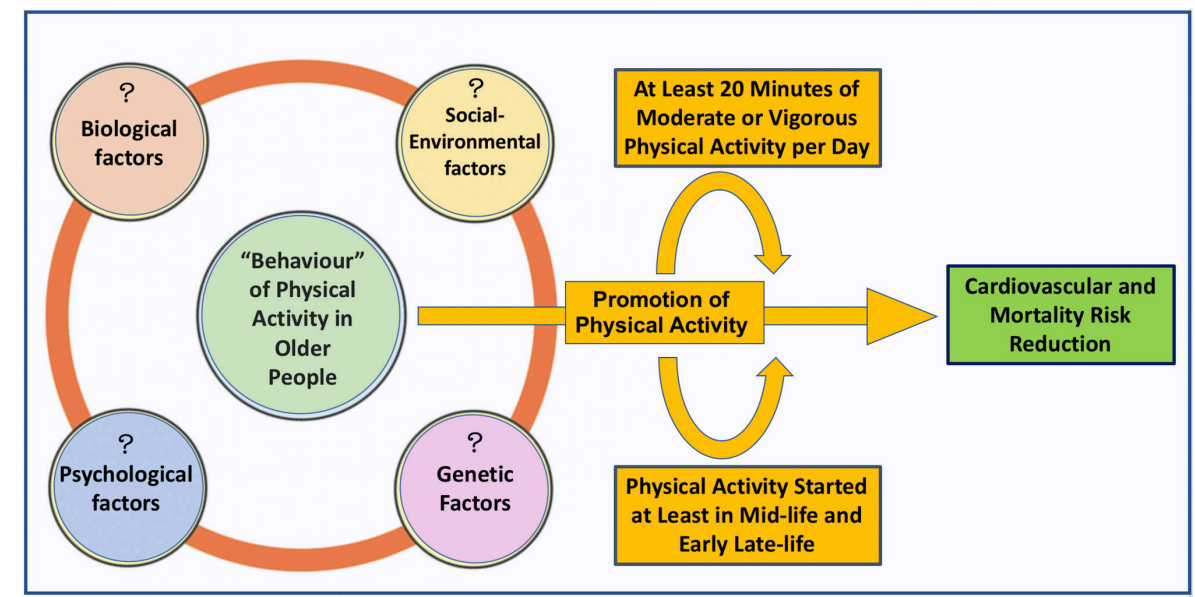

Figure 2 Relationship between 'Behaviour' of Physical Activity, the Promotion of Physical Activity and Physical Activity Level to reduce cardiovascular and mortality risk. 
Kaplan-Meier Failure Estimates by AF Status (HFrEF vs. HFpEF)

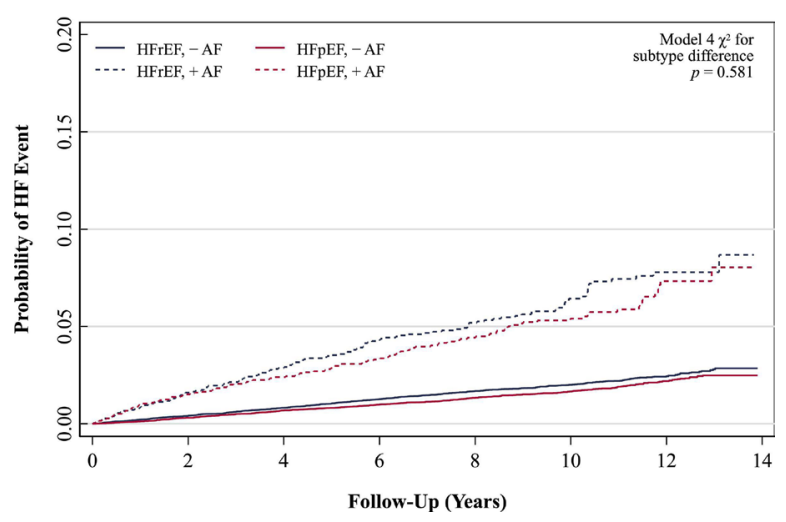

Figure 3 Unadjusted Kaplan-Meier failure curves for HFrEF and HFpEF events in the augmented data set for Lunn-McNeil analysis, with each heart failure subtype stratified by baseline AF status. $A F$, atrial fibrillation; $H F$, heart failure; $H F p E F$, heart failure with preserved ejection fraction; $H F r E F$, heart failure with reduced ejection fraction.

in men with CRTI compared with uninjured men.

In an editorial, Stewart ${ }^{4}$ provides potential explanations for the association between CRTI and long-term adverse cardiovascular outcomes $\mathrm{He}$ points out that there are several unanswered questions including whether the effects of CRTI are the same in women as in men, whether differences in access to care, socioeconomics and race/ethnicity are important and 'most importantly, what interventions can be made to mitigate complications and improve long-term outcomes for wounded service members? We owe it to these young men and women, every one of them a volunteer, who have sacrificed so much for our nations, to answer these questions.'

Another interesting paper in this issue of Heart examines the association between atrial fibrillation (AF) and heart failure with reduced (HFrEF) or preserved ejection fraction $(\mathrm{HFpEF}) .^{5}$ Over 9 years of follow-up of 25787 participants free of baseline HF from the REGARDS (REasons

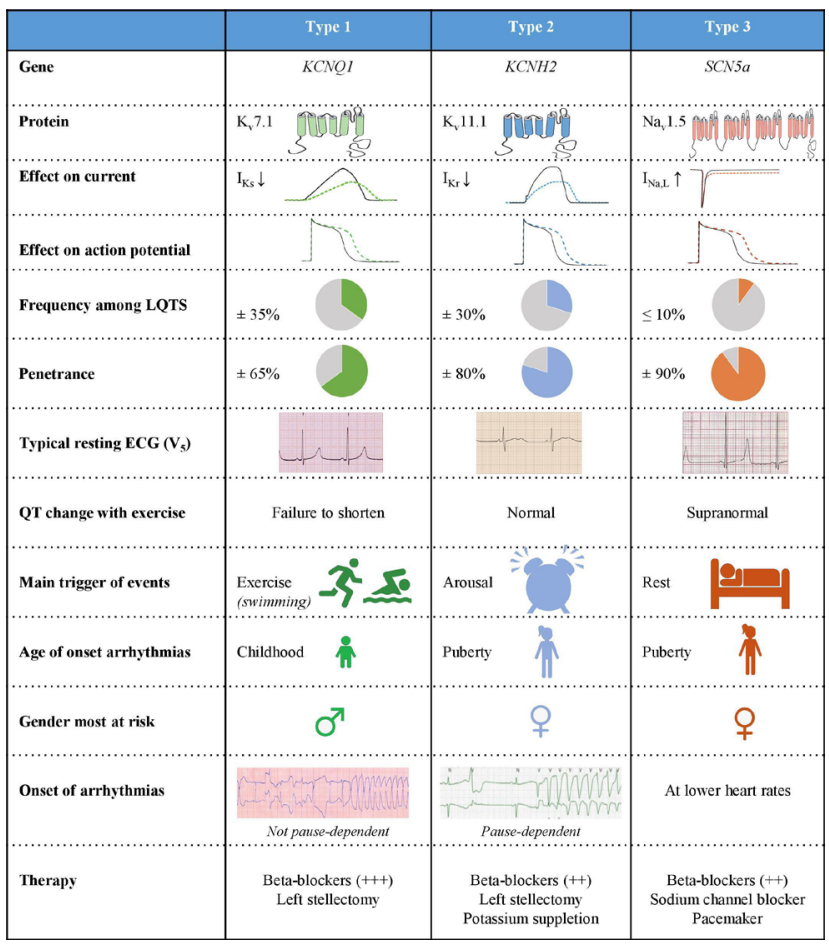

Figure 4 Genotype-phenotype relationship for the three most important subtypes, types 1, 2 and 3 . See text for further explanation. $I_{\mathrm{Kr}^{\prime}}$ rapidly activating delayed rectifier potassium current; $I_{\mathrm{Kr}}$ rapidly activating delayed rectifier potassium current; $I_{\mathrm{Na},{ }^{\prime}}$ late sodium current; LQTS, long QT syndrome ; $\downarrow$, decrease; $\uparrow$, increase; + , therapeutic effect size of $\beta$-blocker therapy. for Geographic And Racial Differences in Stroke) cohort, AF was associated with an increased risk of heart failure events (HR: $1.67,95 \%$ CI 1.38 to 2.01 ) after adjustment for sociodemographics, cardiovascular risk factors and incident coronary heart disease, with no differences in the association of $\mathrm{AF}$ and heart failure between groups with HFpEF or HFrEF (figure 3).

Readers will want to take a look at the two review articles in this issue. The first review article addresses the possible contribution of inflammatory and metabolic factors associated with epicardial adipose tissue to the pathophysiology of HFpEF. ${ }^{6}$ The second review article ${ }^{7}$ summarises the diagnosis and management of congenital long QT syndrome, including a detailed discussion the three main congenital long QT syndrome subtypes (figure 4).

The Education in Heart article in this issue addresses management of cardiovascular disease in the intensive care unit. ${ }^{8}$ The article starts with a high-level overview of the physiology of acute heart failure and cardiogenic shock, including the key concepts of contractility, preload, afterload and electrical conductivity. A general approach to treatment considers cardiac index (CI), systemic vascular resistance index (SVRI) and pulmonary capillary wedge pressure (PCWP) to subclassify cardiogenic shock as classic (low CI, increased SVRI and high PSWP), vasodilatory (high CI, low SVRI and PCWP), euvolemic (low CI, increased SVRI and normal PCWP) and mixed. 'Initial clinical evaluation should adopt an aetiologically directed approach following the CHAMP acronym (acute Coronary syndrome, Hypertension emergency, Arrhythmia, acute Mechanical cause, Pulmonary embolism) with multimodal assessment.' The importance of a multispecialty team approach and optimisation of ventilation, sedation and renal support are emphasised. Finally, options for patients refractory to conventional haemodynamic support include mechanical circulatory support, heart transplantation and end-of-life care.

Funding The authors have not declared a specific grant for this research from any funding agency in the public, commercial or not-for-profit sectors.

\section{Competing interests None declared.}

Patient and public involvement Patients and/ or the public were not involved in the design, or conduct, or reporting, or dissemination plans of this research.

Patient consent for publication Not applicable.

Ethics approval This study does not involve human participants.

Provenance and peer review Commissioned; internally peer reviewed. 
(c) Author(s) (or their employer(s)) 2022. No commercial re-use. See rights and permissions. Published by BMJ.

\section{(A) Check for updates}

To cite Otto CM. Heart 2022;108:325-327.

Heart 2022;108:325-327.

doi:10.1136/heartjnl-2022-320892

\section{ORCID ID}

Catherine M Otto http://orcid.org/0000-0002-05279392

\section{REFERENCES}

1 Barbiellini Amidei C, Trevisan C, Dotto M. Association of physical activity trajectories with major cardiovascular diseases in elderly people. Heart 2022;108:360-6.

2 Fabris E, Sinagra G. Physical activity in older people: better late than never, but better early than late. Heart 2022;108:328-9.

3 Boos CJ, Schofield S, Cullinan P. Association between combat-related traumatic injury and cardiovascular risk. Heart 2022;108:367-74.

4 Stewart IJ. Forever wounds of the forever war. Heart 2022;108:330-1.
5 Nicoli CD, O'Neal WT, Levitan EB. Atrial fibrillation and risk of incident heart failure with reduced versus preserved ejection fraction. Heart 2022;108:353-9.

6 Ayton SL, Gulsin GS, McCann GP. Epicardial adipose tissue in obesity-related cardiac dysfunction. Heart 2022;108:339-44.

7 Wilde AAM, Amin AS, Postema PG. Diagnosis, management and therapeutic strategies for congenital long QT syndrome. Heart 2022;108:332-8.

8 Cook DJ, Webb S, Proudfoot A. Assessment and management of cardiovascular disease in the intensive care unit. Heart 2022;108:397-405. 